\title{
Flow Equivalence and Stochastic Equivalence in G-Networks *
}

\author{
Jean-Michel Fourneau \\ Laboratoire PRISM \\ Univ. de Versailles Saint-Quentin \\ 45 Avenue des Etats-Unis \\ 78000 Versailles, France \\ jmf@prism.uvsq.fr
}

\author{
Erol Gelenbe ${ }^{\dagger}$ \\ Dennis Gabor Chair \\ Dept. of Electrical and Electronic Engineering \\ Imperial College \\ London SW7 2AZ, UK \\ e.gelenbe@imperial.ac.uk
}

\begin{abstract}
G-networks are novel product form queuing networks that, in addition to ordinary customers, contain unusual entities such as "negative customers" which eliminate normal customers, and "triggers" that move other customers from some queue to another. Recently we introduced one more special type of customer, a "reset", which may be sent out by any server at the end of a service epoch, and that will reset the queue to which it arrives into its steady state when that queue is empty. A reset which arrives to a non-empty queue has no effect at all. The sample paths of a system with resets is significantly different from that of a system without resets, because the arrival of a reset to an empty queue will provoke a positive jump in queue length which may be of arbitrarily large size, while ithout resets positive jumps are only of size +1 when a positive customer arrives to a queue. In this paper we review this novel model, and then discuss its traffic equations. We introduce the concept of "stationary equivalence" for queueing models, and of "flow equivalence" for distinct queueing models. We show that the flow equivalence of two G-networks implies that they are also stationary equivalent. We then show that the stationary probability distribution of a G-network with resets is identical to that of a G-nework without resets whose transition probabilities for positive (ordinary) customers has been increased in a specific manner. Our results show that a G-network with resets has the same form of traffic equations and the same joint stationary probability distribution of queue length as that of a G-network without resets.
\end{abstract}

\section{Introduction}

Queueing networks are used to model systems in which a set of customers circulate among servers, possibly waiting in line, then obtaining service, after which they may proceed to another service station or leave the service system altogether after completing a series of service requests. Queueing networks are widely used in industry to represent manufacturing systems, job-shops, the Internet, telephone and telecommunication networks, or other service facilities. Because of their widespread usage and great complexity, such systems have been studied abundantly over the last one hundred years, and over the last thirty years mathematical methods and software tools have been developed to analyse and predict their behaviour. Software tools that have been successfully used in this area include simulation packages, analytical solvers, and approximate numerical solvers.

\footnotetext{
${ }^{*}$ This work is supported by the UK EPSRC (Engineering and Physical Sciences Research Council) under grant GR/S52360/01, and by the European Union Project FP6 506602 SAPAD

${ }^{\dagger}$ Contact author.
} 
Typically, the time dependent behaviour of such systems is characterised by very large systems of differential-difference equations which describe the manner in which the probability distribution of the queue lengths and of other quantities of interest (such as the stage of service occupied by each customer in the network), evolve over time. These state equations are relations concerning the change over time of the joint probability distributions of network state. The number of equations concerned can be countably infinite if the network is "open", i.e. if the network exchanges customers with the oustide world. Although the number is finite when the system is "closed", it will grow very rapidly with the number of customers and service stations even in this case.

Because such systems can be very complex, mathematical methods have been developed to study their steady state or long term behaviour $[1,2]$. Such methods can be used to obtain exact algebraic expressions in terms of significant parameters such as service time distributions, customer arrival rates, and the transition probabilities of customers from one queue to another, which simplify the computation of many of the measures of interest. In particular, certain classes of queueing networks have been shown to have "product form" which is a remarkable property stating that, in steady-state, the joint probability distribution of system state can be expressed as the product of its marginal distributions. Such a result, when it is available, considerably simplifies the exact analysis of complex queueing networks and aids in the computation of performance measures for large computer systems and networks.

G-networks were introduced in the last decade so as to model the behaviour of queueing networks in which, in addition to customers which request and receive service, we can also model the behaviour of control actions such as the removal of certain customers using "negative customers", and the displacement of customers from one queue to another before they receive service using "triggers" $[3,4,5,6,7,13]$. For instance, negative customers can be used to model flow control in a system, while triggers can be used to model actions resulting in load balancing. Multiple classes of customers are used in queueing networks to model groups of customers which have distinct probabilistic characteristics, and multiple class versions of G-networks have been developed, and solution techniques for state dependent arrival and service processes have been derived $[10,11,12]$. Formal mathematical relationships between G-networks and stochastic neural networks (RNN) are described in $[8,9,16]$.

"Reset" customers introduced in [15] are a recent extension to G-networks. Resets are special signals that can be sent from some queue in the network to any other queue; they may also arrive from outside the network. A normal customer leaving a queue can become a reset and then be sent to some other queue. When a reset arrives at a queue:

- 1) If the queue is not empty, the reset has no effect and is immediately lost.

- 2) If the queue is empty then the queue length is reset to a random queue length whose distribution is identical to the stationary distribution at that queue.

In [15] we have shown that this model has product form solution under assumptions of Poisson external arrivals, exponential service times for ordinary customers, and Markovian customer movement between queues including transitions of ordinary customers which become negative customers, triggers or resets.

In an "ordinary" G-network, the sample paths describing the manner in which the Ndimensional state representing the number of customers in each of the $\mathrm{N}$ queues only has positive jumps of size one, resulting from the arrival of some customer at a queue. However, the arrival of a reset customer to a queue results in a random sized positive jump in the sample path. Thus 
the sample paths of queueing models with resets have jumps which are distinct from the those of models without resets. Despite this difference, we will show that these two distinct models are equivalent in an interesting and non-trivial manner.

\section{$1.1 \quad$ G-Networks with Resets}

The G-network model we consider has $N$ service centres and customer queues. Each queue $j$ has independent and identically exponentially distributed service times with rates $\mu_{j}, j=1, \ldots, N$. Queue $j$ receives positive customers from outside the network according to a Poisson process of rate $\lambda_{j}^{+}$. Positive customers are the ordinary queuing network customers which receive service at the various queues. In addition to the usual customers, the network also contains "signals". These signals arrive to the queue $i$ from outside the network in a Poisson stream with parameter $\lambda_{i}^{-}$. Both positive customers and signals can also arrive to a queue from some other queue.

In this paper, signals can be one of three special types of customers:

- Negative customers which destroy an existing customer,

- Triggers which move a customer from some queue to another,

- Resets which are the new type of customers introduced in [15].

When an ordinary or "positive" customer leaves a queue at the end of a service epoch, it will either leave the network, enter another queue as an ordinary customer, or arrive at another queue as a signal. Customer transitions are defined by the probability matrices $P^{+}=\left[P_{i j}^{+}\right]$ for a positive customer leaving a queue and joining another queue as a positive customer, or $P^{-}=\left[P_{i j}^{-}\right]$for a normal customer leaving a queue heading into another queue as a signal. Positive customers to a queue either arrive from outside the network, or they arrive from other queues. In the latter case, they are either positive customers which have been triggered (by signals) from other queues, or they are positive customers which have completed service at some other queue. Signals, on the other hand, can either arrive from outside the network, or they result from the change of a positive customer leaving a queue to become a signal at another queue. With probability $d_{i}$, a positive customer leaves the network after completing service at queue $i$ :

$$
d_{i}=1-\sum_{j=1}^{N}\left[P_{i j}^{+}+P_{i j}^{-}\right] .
$$

The effect of signals is described as follows:

- When a signal arrives from some queue $i$ to some queue $j$ with probability $P_{i j}^{-}$:

1. If queue $j$ is non-empty, the signal triggers a customer to move instantaneously to some other queue with probability $\alpha_{i, j}$, and with probability $\left[1-\alpha_{i, j}\right]$ the signal has no effect. With probability $Q_{j k}$ the customer which has been triggered moves to some other queue $k$, or leaves the network with probability $Q_{j, N+1}$. Note that for any $i$, we have:

$$
Q_{i, N+1}=1-\sum_{j=1}^{N} Q_{i, j} .
$$

2. If queue $j$ is empty, the signal will create a random batch of $Y_{i j}$ customers at queue $j$ with probability $\beta_{i, j}$. With probability $\left[1-\beta_{i, j}\right]$ the signal has no effect. The distribution of $Y_{i j}$ is denoted by $\operatorname{Pr}\left[Y_{i j}=x_{j}\right]=\tau_{j}\left(x_{j}\right)$. 
- In all cases, after a signal has acted upon the queue to which it arrives, it vanishes (i.e., signals do not queue or receive service).

When a signal arrives from outside the network to some queue $j$, we replace the probabilities $\alpha_{i, j}, \beta_{i, j}$ by $\alpha_{0, j}, \beta_{0, j}$.

\subsection{Traffic equations and stationary solution for G-networks}

For each queue $i$, the traffic equations are used to describe the arrival rate $\Lambda_{i}^{+}$of positive customers, and the arrival rate $\Lambda_{i}^{-}$of signals (including resets, negative customers and triggers).

Notation Consider the following system of equations, which can be interpreted as the traffic equations for the G-network with resets:

$$
\begin{aligned}
\Lambda_{i}^{+}= & \lambda_{i}^{+}+\sum_{j=1}^{N} \mu_{j} \rho_{j} P_{j i}^{+}+\sum_{j=1}^{N} \lambda_{j}^{-} \rho_{j} \alpha_{0, j} Q_{j, i} \\
& +\sum_{j=1}^{N} \sum_{k=1}^{N} \mu_{j} \rho_{j} P_{j k}^{-} \rho_{k} \alpha_{j, k} Q_{k, i} \\
& +\lambda_{i}^{-} \beta_{0, i}+\sum_{j=1}^{N} \mu_{j} \rho_{j} P_{j i}^{-} \beta_{i, j}
\end{aligned}
$$

and:

$$
\Lambda_{i}^{-}=\lambda_{i}^{-} \alpha_{0, i}+\sum_{j=1}^{N} \mu_{j} \rho_{j} P_{j i}^{-} \alpha_{j, i}
$$

where:

$$
\rho_{i}=\frac{\Lambda_{i}^{+}}{\mu_{i}+\Lambda_{i}^{-}}
$$

To render the notation more compact, let us define the following vectors:

$$
\begin{gathered}
\vec{\Lambda}^{+} \text {with elements }\left[\Lambda_{i}^{+}\right], \\
\vec{\lambda}^{+} \text {with elements }\left[\lambda_{i}^{-}\right], \\
\vec{\Lambda}^{-} \text {with elements }\left[\Lambda_{i}^{-}\right], \\
\vec{\lambda}^{-} \text {with elements }\left[\lambda_{i}^{-}\right], \\
\alpha_{0}=\left(\alpha_{0,1}, \ldots, \alpha_{0, N}\right), \beta_{0}=\left(\beta_{0,1}, \ldots, \beta_{0, N}\right) .
\end{gathered}
$$

Also, let $P^{+}, P^{-}, Q$, be matrices with elements $P^{+}[i, j], P^{-}[i, j]$ and $Q[i, j]$, respectively, and let $A$ and $B$ be the $N \times N$ matrices with elements $\alpha_{i j}$ and $\beta_{i j}$, respectively, for $1 \leq i, j \leq N$. Remark Notice that a network $\hat{\mathcal{G}}$ without resets is equivalent to a network with resets if the matrix $B$ is identically 0 , and if the vector $\beta_{0}$ is also identically zero.

We will write $X * Y$ to represent the matrix obtained by the element by element product of the two $N \times N$ matrices $X$ and $Y$, and let $u * v$ be the element by element product of $N$-vectors $u$ and $v$.

Lemma 1 The traffic equations for the G-network with resets (3), (4) can be written as:

$$
\vec{\Lambda}^{+}=\lambda^{+}+\lambda^{-} * \beta_{0}+\vec{\Lambda}^{+}\left\{F P^{+}+[1-F] Q+F P^{-} * B\right\},
$$




$$
\vec{\Lambda}^{-}=\lambda^{+} * \alpha_{0}+\vec{\Lambda}^{+} F P^{-} * A,
$$

where $F$ is the $N \times N$ diagonal matrix whose elements are given by:

$$
\begin{aligned}
F_{i, i} & =\frac{\mu_{i}}{\mu_{i}+\Lambda_{i}^{-}} \\
F_{i, j} & =0, i \neq j .
\end{aligned}
$$

Proof We can write (3) as:

$$
\Lambda_{i}^{+}=\lambda_{i}^{+}+\sum_{j=1}^{N} \mu_{j} \rho_{j} P_{j i}^{+}+\sum_{j=1}^{N} \Lambda_{j}^{-} \rho_{j} Q_{j, i}+\lambda_{i}^{-} \beta_{0, i}+\sum_{j=1}^{N} \mu_{j} \rho_{j} P_{j i}^{-} \beta_{i, j}
$$

which becomes (6) when we use vector notation. On the other hand, by substituting (5) in (4) we obtain:

$$
\Lambda_{i}^{-}=\lambda_{i}^{-} \alpha_{0, i}+\sum_{j=1}^{N} \Lambda_{j}^{+} \frac{\mu_{j}}{\mu_{j}+\Lambda_{j}^{-}} P_{j i}^{-} \alpha_{j, i}
$$

which becomes (7) when it is written in vector notation. Q.E.D.

The next result establishes that the stationary solution to a G-network with resets has product form. The result was first proved in [15]; however we present the proof again in the Appendix at the end of this paper in order to provide the reader with a self-contained presentation.

Theorem 1 Consider the G-network, denoted by $\mathcal{G}$, which is specified using:

- (i) The matrices $P^{-}, P^{+}, Q, A, B$, and vectors $\alpha_{0}, \beta_{0}$,

- (ii) The external arrival rates $\lambda_{i}^{+}$and $\lambda_{i}^{-}$, and

- (iii) The reset probability distribution:

$$
\begin{aligned}
\tau_{j}\left(x_{j}\right) & =\left(1-\rho_{j}\right)\left(\rho_{j}\right)^{\left(x_{j}-1\right)}, x_{j}>0 \\
\tau_{j}(0) & =0 .
\end{aligned}
$$

Let $\left(X_{1}(t), \ldots, X_{N}(t)\right)$ represent the queue length vector at time $t$ for the system $\mathcal{G}$ with $N$ servers, and let $\pi(\vec{x})$ be the stationary probability, if it exists, that the network state is $\vec{x}=$ $\left(x_{1}, \ldots, x_{N}\right)$.

If equations (3), (4), (5) have non-negative solutions such that all the $\rho_{i}<1$, for $i=$ $1, \ldots, N$, then the stationary distribution for $\mathcal{G}$ exists and has the product form:

$$
\pi(\vec{x})=\prod_{i=1}^{N} \pi_{i}\left(x_{i}\right)
$$

where the $\pi_{i}\left(x_{i}\right)=\left(\rho_{i}\right)^{x_{i}}\left(1-\rho_{i}\right)$ are the marginal probabilities of queue length at each of the queues. 


\section{Flow Equivalence and Stationary Equivalence of Queueing Networks}

Let us first formalize two properties of queueing networks, the first one being very common in queueing network models, while the second one is specific to G-networks:

- The first property will be called stationary equivalence (SE): we will say that two queueing networks with the same number of queues are SE if the stationary joint queue length distributions of both systems are identical.

- The second property will be called flow equivalence (FE): two queueing models which have the same number of servers are said to be $\mathbf{F E}$ if (i) the steady-state rate at which positive customers arrive at each queue are identical in each of the two networks, and (ii) the steady-state rate at which signals arrive at each queue are identical.

More formally, the two definitions are as follows.

Definition 1 Let $X(t)=\left(X_{1}(t), \ldots, X_{N}(t)\right), x(t)=\left(x_{1}, \ldots, x_{N}(t)\right)$ be the vectors of queue lengths for two queueing networks $\mathcal{Q}$ and $\hat{\mathcal{Q}}$ each having $N$ service centres. We will say that $Q$ and $\hat{Q}$ are $\mathbf{S E}$ if and only if for all $N$-vectors $\vec{x}$ with non-negative integer elements:

$$
\lim _{t \rightarrow \infty} P[X(t)=\vec{x}]=\lim _{t \rightarrow \infty} P[x(t)=\vec{x}] .
$$

Definition 2 Let $\Lambda_{i}^{+}(Q), \Lambda_{i}^{-}(Q)$ denote the arrival rates of positive customers and signals to the $i$-th queue of $G$-network $Q$. We will say that two G-networks $\mathcal{Q}$ and $\mathcal{Q}^{*}$ with $N$ service centres are $\mathbf{F E}$ if and only if $\Lambda_{i}^{+}(\mathcal{Q})=\Lambda_{i}^{+}\left(\mathcal{Q}^{*}\right)$, and $\Lambda_{i}^{-}(\mathcal{Q})=\Lambda_{i}^{-}\left(\mathcal{Q}^{*}\right)$ for all $i=1, \ldots, N$.

The next result follows directly from Theorem 1 and the definitions we have just given.

Theorem 2 If the $G$-networks $\mathcal{G}$ and $\mathcal{G}^{*}$ with the same number $N$ of service centers are $\mathbf{F E}$, and have identical (exponential) service times distributions centre by centre (say with service rates in both systems given by $\left.\mu_{1}, \ldots \mu_{N}\right)$, then they are also $\mathbf{S E}$.

\subsection{Equivalence of Distinct Models}

The next theorem says that two G-networks, one with resets and the other without reset customers, are stochastically equivalent provided that the parameter set of the system without resets is obtained in a specific manner from the parameter set of the network with resets. Specifically, the probabilites associated with transitions of positive customers for the network without resets are greater than that of the network with resets in a specific manner.

Theorem 3 Consider two $G$-networks $\mathcal{G}$ and $\hat{\mathcal{G}}$ with the same number $N$ of service centers, and identical exponential service time distributions with rates $\mu_{1}, \ldots \mu_{N} . \mathcal{G}$ has reset customers and parameter set $P^{-}, P^{+}, Q, A, B, \alpha_{0}, \beta_{0}$, with reset probability distribution:

$$
\begin{aligned}
\tau_{j}\left(x_{j}\right) & =\left(1-\rho_{j}\right)\left(\rho_{j}\right)^{\left(x_{j}-1\right)}, x_{j}>0 \\
\tau_{j}(0) & =0 .
\end{aligned}
$$

where $\rho_{j}=\Lambda_{j}^{+} /\left[\mu_{j}+\Lambda_{j}^{-}\right]$. The network $\hat{\mathcal{G}}$ does not have reset customers and is defined by the parameter set $P_{n}^{-}, P_{n}^{+}, Q_{n}, A_{n}, \alpha_{0}^{n}$, where: 
- $A_{n}=A$ and the vectors $\alpha_{0}$ and $\alpha_{0}^{n}$ are identical,

- $P_{n}^{+}=P^{+}$,

- The vector $\beta_{0}$ need not be specified for $\hat{\mathcal{G}}$, or alternatively we may just say that it has the value $\beta_{0}^{n}=0$.

- Finally, $P_{n}^{-}=P^{+}+P^{-} * B$.

Then $\mathcal{G}$ and $\hat{\mathcal{G}}$ are $\mathbf{S E}$.

Proof We will first prove that $\hat{\mathcal{G}}$ and $\mathcal{G}$ are $\mathbf{F E}$, and then invoke Theorem 2 to show that they are also SE.

To prove that they are $\mathbf{F E}$, notice that by Lemma 1, the traffic equations for $\mathcal{G}$ may be written as:

$$
\vec{\Lambda}^{-}=\lambda^{+} * \alpha_{0}+\vec{\Lambda}^{+} F P^{-} * A,
$$

which is unchanged from (7), while (6) is identical to:

$$
\vec{\Lambda}^{+}=\lambda^{+}+\lambda^{-} * \beta_{0}+\vec{\Lambda}^{+}\left\{F\left[P^{+}+P^{-} * B\right]+[1-F] Q\right\},
$$

which, by substituting $P_{n}^{-}=P^{+}+P^{-} * B$ can be written as:

$$
\vec{\Lambda}^{+}=\lambda^{+}+\lambda^{-} * \beta_{0}+\vec{\Lambda}^{+}\left\{F P_{n}^{-}+[1-F] Q\right\} .
$$

Now note that (19) and (17) are the traffic equations for $\hat{\mathcal{G}}$, so that $\mathcal{G}$ and $\hat{\mathcal{G}}$ are $\mathbf{F E}$ and are therefore also $\mathbf{S E}$ by Theorem 2, completing the proof. Q.E.D

\section{Conclusions}

In this paper we have reviewed G-network queueing models with the recent extension of "reset" customers. We have recalled the product form result for the model with "resets" and then discussed their traffic equations. We also introduce the concept of "stationary equivalence" for queueing models, and of "flow equivalence" for distinct queueing models. We show that the flow equivalence of two G-networks implies that they are also stationary equivalent.

Based on these two concepts of equivalence, we show that the stationary probability distribution of a G-network with resets is identical to that of a G-nework without resets whose transition probabilities for positive (ordinary) customers has been increased in a manner which depends on the reset transition probabilities. The proof is based on showing that a G-network with resets has traffic equations which have the same general form of those for a G-network without resets. Future work will consider extensions to more complex state transition rules and to other novel product form results.

\section{References}

[1] Gelenbe, E., Mitrani I., "Analysis and Synthesis of Computer Systems", Academic Press, New York and London, 1980.

[2] Ferrari D., Serazzi G., Zeigner A., "Measurement and Tuning of Computer Systems", Prentice-Hall, Englewood Cliffs, N.J., 1983. 
[3] E. Gelenbe "Queuing networks with negative and positive customers", Journal of Applied Probability, Vol. 28, pp 656-663 (1991).

[4] E. Gelenbe, P. Glynn, K. Sigman "Queues with negative arrivals", Journal of Applied Probability, Vol. 28, pp 245-250, 1991.

[5] E. Gelenbe, M. Schassberger "Stability of product form G-Networks", Probability in the Engineering and Informational Sciences, 6, pp 271-276, 1992.

[6] E. Gelenbe "G-networks with instantaneous customer movement", Journal of Applied Probability, 30 (3), 742-748, 1993.

[7] Erol Gelenbe "G-Networks with signals and batch removal", Probability in the Engineering and Informatonal Sciences, 7, pp 335-342, 1993.

[8] E. Gelenbe "Learning in the recurrent random network", Neural Computation, 5, pp 154164, 1993.

[9] E. Gelenbe "G-networks: An unifying model for queuing networks and neural networks," Annals of Opns. Res., Vol. 48, No. 1-4, pp 433-461, 1994.

[10] J.M. Fourneau, E. Gelenbe, R. Suros "G-networks with multiple classes of positive and negative customers," Theoretical Computer Science Vol. 155 (1996), pp.141-156.

[11] E. Gelenbe, A. Labed "G-networks with multiple classes of signals and positive customers", European J. Opns. Res., Vol. 108 (2), pp. 293-305, July 1998.

[12] M. Pinedo, X. Chao, M. Miyazawa "Queuing Networks: Customers, Signals and Product Form Solutions", J. Wiley, Chichester and New York, 1999.

[13] J.R. Artalejo "G-networks: a versatile approach for work removal in queuing networks," European J. Opns. Res., Vol. 126, 233-249, 2000.

[14] Feature Issue on G-networks, European J. Op. Res., Vol. 126, 2000.

[15] E. Gelenbe, J.M. Fourneau "G-Networks with resets", Performance Evaluation, Vol. 49, pp. 179-192, 2002, also in Proc. IFIP WG 7.3/ACM-SIGMETRICS Performance '02 Conf., Rome, Italy, October 2002.

[16] E. Gelenbe, K. Hussain "Learning in the multiple class random neural network", IEEE Trans. on Neural Networks, Vol. 13 (6), 1257-1267, 2002.

\section{Appendix: Proof of Theorem 1}

For the sake of completeness, this Appendix contains the proof of Theorem 1 which was given in [15], which establishes that (13) satisfies the global balance equations (21). We write:

$$
\pi(\vec{x})=\lim _{t \rightarrow \infty} \operatorname{Pr}\left[\left(X_{1}(t), \ldots, X_{N}(t)\right)=\vec{x}\right] .
$$

amd use $\overrightarrow{e_{i}}$ to denote the $N$-vector which is 0 everywhere, except in position $i$ where it has the value $1: \overrightarrow{e_{i}}=(0, . ., 1, \ldots, 0)$. The Chapman-Kolmogorov equations for the G-network with 
resets in steady-state, i.e. the global balance equations, can be written as:

$$
\begin{aligned}
\pi(\vec{x}) \sum_{i=1}^{N}[ & \left.\lambda_{i}^{+}+\left(\mu_{i}+\lambda_{i}^{-} \alpha_{0, i}\right) 1_{\left\{x_{i}>0\right\}}+\lambda_{i}^{-} 1_{\left\{x_{i}=0\right\}} \beta_{0, i}\right]= \\
& \quad \sum_{i=1}^{N} \lambda_{i}^{+} \pi\left(\vec{x}-\overrightarrow{e_{i}}\right) 1_{\left\{x_{i}>0\right\}} \\
& +\sum_{i=1}^{N} \mu_{i} \pi\left(\vec{x}+\overrightarrow{e_{i}}\right) d_{i} \\
& +\sum_{i=1}^{N} \lambda_{i}^{-} \alpha_{0, i} Q_{i, N+1} \pi\left(\vec{x}+\overrightarrow{e_{i}}\right) \\
& +\sum_{i=1}^{N} \sum_{j=1}^{N} \lambda_{i}^{-} \alpha_{0, i} Q_{i, j} \pi\left(\vec{x}+\overrightarrow{e_{i}}-\overrightarrow{e_{j}}\right) 1_{\left\{x_{j}>0\right\}} \\
& +\sum_{i=1}^{N} \sum_{j=1}^{N} \mu_{i} \pi\left(\vec{x}+\overrightarrow{e_{i}}-\overrightarrow{e_{j}}\right) P_{i j}^{+} 1_{\left\{x_{j}>0\right\}} \\
& +\sum_{i=1}^{N} \sum_{j=1}^{N} \mu_{i} \pi\left(\vec{x}+\overrightarrow{e_{i}}+\overrightarrow{e_{j}}\right) P_{i j}^{-} Q_{j, N+1} \alpha_{i, j} \\
& +\sum_{i=1}^{N} \sum_{j=1}^{N} \sum_{k=1}^{N} \mu_{i} \pi\left(\vec{x}+\overrightarrow{e_{i}}+\overrightarrow{e_{j}}-\overrightarrow{e_{k}}\right) P_{i j}^{-} Q_{j, k} \alpha_{i, j} 1_{\left\{x_{k}>0\right\}} \\
& +\sum_{i=1}^{N} \sum_{j=1}^{N} \mu_{i} \pi\left(\vec{x}+\overrightarrow{e_{i}}\right) P_{i j}^{-}\left(1-\alpha_{i, j}\right) 1_{\left\{x_{j}>0\right\}} \\
& +\sum_{i=1}^{N} \sum_{j=1}^{N} \mu_{i} \pi\left(\vec{x}+\overrightarrow{e_{i}}-x_{j} \overrightarrow{e_{j}}\right) P_{i j}^{-} \beta_{i, j} 1_{\left\{x_{j}>0\right\}} \tau_{j}\left(x_{j}\right) \\
& +\sum_{i=1}^{N} \lambda_{i}^{-} \beta_{0, i} \pi\left(\vec{x}-x_{i} \overrightarrow{e_{i}}\right) 1_{\left\{x_{i}>0\right\}} \tau_{i}\left(x_{i}\right) \\
& +\sum_{i=1}^{N} \sum_{j=1}^{N} \mu_{i} \pi\left(\vec{x}+\overrightarrow{e_{i}}\right) P_{i j}^{-}\left(1-\beta_{i, j}\right) 1_{\left\{x_{j}=0\right\}}
\end{aligned}
$$

We start by by substituting (13) in (21), and dividing both sides by the resulting expression by $\left(\rho_{i}\right)^{x_{i}}$. This results in:

$$
\begin{aligned}
\sum_{i=1}^{N}\left[\lambda_{i}^{+}+\right. & \left.\left(\mu_{i}+\lambda_{i}^{-} \alpha_{0, i}\right) 1_{\left\{x_{i}>0\right\}}+\lambda_{i}^{-} 1_{\left\{x_{i}=0\right\}} \beta_{0, i}\right]= \\
& \sum_{i=1}^{N} \lambda_{i}^{+} \frac{1}{\rho_{i}} 1_{\left\{x_{i}>0\right\}} \\
& +\sum_{i=1}^{N} \mu_{i} \rho_{i} d_{i} \\
& +\sum_{i=1}^{N} \lambda_{i}^{-} \alpha_{0, i} Q_{i, N+1} \rho_{i} \\
& +\sum_{i=1}^{N} \sum_{j=1}^{N} \lambda_{i}^{-} \alpha_{0, i} Q_{i, j} \frac{\rho_{i}}{\rho_{j}} 1_{\left\{x_{j}>0\right\}} \\
& +\sum_{i=1}^{N} \sum_{j=1}^{N} \mu_{i} \frac{\rho_{i}}{\rho_{j}} P_{i j}^{+} 1_{\left\{x_{j}>0\right\}} \\
& +\sum_{i=1}^{N} \sum_{j=1}^{N} \mu_{i} \rho_{i} \rho_{j} P_{i j}^{-} Q_{j, N+1} \alpha_{i, j} \\
& +\sum_{i=1}^{N} \sum_{j=1}^{N} \sum_{k=1}^{N} \mu_{i} \frac{\rho_{i} \rho_{j}}{\rho_{k}} P_{i j}^{-} Q_{j, k} \alpha_{i, j} 1_{\left\{x_{k}>0\right\}} \\
& +\sum_{i=1}^{N} \sum_{j=1}^{N} \mu_{i} \rho_{i} P_{i j}^{-}\left(1-\alpha_{i, j}\right) 1_{\left\{x_{j}>0\right\}} \\
& +\sum_{i=1}^{N} \sum_{j=1}^{N} \mu_{i} \frac{\rho_{i}}{\left(\rho_{j}\right)^{x_{j}}} P_{i j}^{-} \beta_{i, j} 1_{\left\{x_{j}>0\right\}} \tau_{j}\left(x_{j}\right) \\
& +\sum_{i=1}^{N} \frac{\lambda_{i}^{-} \beta_{0, i}}{\rho_{i}^{x_{i}}} 1_{\left\{x_{i}>0\right\}} \tau_{i}\left(x_{i}\right) \\
& +\sum_{i=1}^{N} \sum_{j=1}^{N} \mu_{i} \rho_{i} P_{i j}^{-}\left(1-\beta_{i, j}\right) 1_{\left\{x_{j}=0\right\}}
\end{aligned}
$$

which, using $\tau_{j}\left(x_{j}\right)$ simplifies to:

$$
\begin{aligned}
\sum_{i=1}^{N}\left[\lambda_{i}^{+}+\right. & \left.\left(\mu_{i}+\lambda_{i}^{-} \alpha_{0, i}\right) 1_{\left\{x_{i}>0\right\}}+\lambda_{i}^{-} 1_{\left\{x_{i}=0\right\}} \beta_{0, i}\right]= \\
& \sum_{i=1}^{N} \lambda_{i}^{+} \frac{1}{\rho_{i}} 1_{\left\{x_{i}>0\right\}} \\
& +\sum_{i=1}^{N} \mu_{i} \rho_{i} d_{i} \\
& +\sum_{i=1}^{N} \lambda_{i}^{-} \alpha_{0, i} Q_{i, N+1} \rho_{i} \\
& +\sum_{i=1}^{N} \sum_{j=1}^{N} \lambda_{i}^{-} \alpha_{0, i} Q_{i, j} \frac{\rho_{i}}{\rho_{j}} 1_{\left\{x_{j}>0\right\}} \\
& +\sum_{i=1}^{N} \sum_{j=1}^{N} \mu_{i} \frac{\rho_{i}}{\rho_{j}} P_{i j}^{+} 1_{\left\{x_{j}>0\right\}} \\
& +\sum_{i=1}^{N} \sum_{j=1}^{N} \mu_{i} \rho_{i} \rho_{j} P_{i j}^{-} Q_{j, N+1} \alpha_{i, j} \\
& +\sum_{i=1}^{N} \sum_{j=1}^{N} \sum_{k=1}^{N} \mu_{i} \frac{\rho_{i} \rho_{j}}{\rho_{k}} P_{i j}^{-} Q_{j, k} \alpha_{i, j} 1_{\left\{x_{k}>0\right\}} \\
& +\sum_{i=1}^{N} \sum_{j=1}^{N} \mu_{i} \rho_{i} P_{i j}^{-}\left(1-\alpha_{i, j}\right) 1_{\left\{x_{j}>0\right\}} \\
& +\sum_{i=1}^{N} \sum_{j=1}^{N} \mu_{i} \frac{\rho_{i}}{\rho_{j}}\left(1-\rho_{j}\right) P_{i j}^{-} \beta_{i, j} 1_{\left\{x_{j}>0\right\}} \\
& +\sum_{i=1}^{N} \lambda_{i}^{-} \beta_{0, i}\left[\frac{1}{\rho_{i}}-1\right] 1_{\left\{x_{i}>0\right\}} \\
& +\sum_{i=1}^{N} \sum_{j=1}^{N} \mu_{i} \rho_{i} P_{i j}^{-}\left(1-\beta_{i, j}\right) 1_{\left\{x_{j}=0\right\}}
\end{aligned}
$$


or better still, to:

$$
\begin{aligned}
\sum_{i=1}^{N}\left[\lambda_{i}^{+}+\right. & \left.\left(\mu_{i}+\lambda_{i}^{-} \alpha_{0, i}\right) 1_{\left\{x_{i}>0\right\}}+\lambda_{i}^{-} 1_{\left\{x_{i}=0\right\}} \beta_{0, i}\right]= \\
& \sum_{i=1}^{N} \lambda_{i}^{+} \frac{1}{\rho_{i}} 1_{\left\{x_{i}>0\right\}} \\
& +\sum_{i=1}^{N} \mu_{i} \rho_{i} d_{i} \\
& +\sum_{i=1}^{N} \lambda_{i}^{-} \alpha_{0, i} Q_{i, N+1} \rho_{i} \\
& +\sum_{i=1}^{N} \sum_{j=1}^{N} \lambda_{i}^{-} \alpha_{0, i} Q_{i, j} \frac{\rho_{i}}{\rho_{j}} 1_{\left\{x_{j}>0\right\}} \\
& +\sum_{i=1}^{N} \sum_{j=1}^{N} \mu_{i} \frac{\rho_{i}}{\rho_{j}} P_{i j}^{+} 1_{\left\{x_{j}>0\right\}} \\
& +\sum_{i=1}^{N} \sum_{j=1}^{N} \mu_{i} \rho_{i} \rho_{j} P_{i j}^{-} Q_{j, N+1} \alpha_{i, j} \\
& +\sum_{i=1}^{N} \sum_{j=1}^{N} \sum_{k=1}^{N} \mu_{i} \frac{\rho_{i} \rho_{j}}{\rho_{k}} P_{i j}^{-} Q_{j, k} \alpha_{i, j} 1_{\left\{x_{k}>0\right\}} \\
& +\sum_{i=1}^{N} \sum_{j=1}^{N} \mu_{i} \rho_{i} P_{i j}^{-}\left(1-\alpha_{i, j}\right) 1_{\left\{x_{j}>0\right\}} \\
& +\sum_{i=1}^{N} \sum_{j=1}^{N} \mu_{i} \frac{\rho_{i}}{\rho_{j}} P_{i j}^{-} \beta_{i, j} 1_{\left\{x_{j}>0\right\}} \\
& -\sum_{i=1}^{N} \sum_{j=1}^{N} \mu_{i} \rho_{i} P_{i j}^{-} \beta_{i, j} 1_{\left\{x_{j}>0\right\}} \\
& +\sum_{i=1}^{N} \frac{\lambda_{i}^{-} \beta_{0, i}}{\rho_{i}} 1_{\left\{x_{i}>0\right\}} \\
& -\sum_{i=1}^{N} \lambda_{i}^{-} \beta_{0, i} 1_{\left\{x_{i}>0\right\}} \\
& +\sum_{i=1}^{N} \sum_{j=1}^{N} \mu_{i} \rho_{i} P_{i j}^{-}\left(1-\beta_{i, j}\right) 1_{\left\{x_{j}=0\right\}}
\end{aligned}
$$

¿From (3) and (24), it follows that $\sum_{i=1}^{N} \Lambda_{i}^{+} 1_{\left\{x_{i}>0\right\}}=\rho_{i}([1]+[4]+[5]+[7]+[9]+[11])$ where the numbers in brackets correspond to the terms as numbered in equation (24). Using also the relationship (5) which is $\rho_{i}=\frac{\Lambda_{i}^{+}}{\mu_{i}+\Lambda_{i}^{-}}$, we can replace (24) by:

$$
\begin{aligned}
\sum_{i=1}^{N}\left[\lambda_{i}^{+}+\right. & \left.\left(\mu_{i}+\lambda_{i}^{-} \alpha_{0, i}\right) 1_{\left\{x_{i}>0\right\}}+\lambda_{i}^{-} 1_{\left\{x_{i}=0\right\}} \beta_{0, i}\right]= \\
& \sum_{i=1}^{N}\left[\mu_{i}+\Lambda_{i}^{-}\right] 1_{\left\{x_{i}>0\right\}} \\
& +\sum_{i=1}^{N} \mu_{i} \rho_{i} d_{i} \\
& +\sum_{i=1}^{N} \lambda_{i}^{-} \alpha_{0, i} Q_{i, N+1} \rho_{i} \\
& +\sum_{i=1}^{N} \sum_{j=1}^{N} \mu_{i} \rho_{i} \rho_{j} P_{i j}^{-} Q_{j, N+1} \alpha_{i, j} \\
& +\sum_{i=1}^{N} \sum_{j=1}^{N} \mu_{i} \rho_{i} P_{i j}^{-}\left(1-\alpha_{i, j}\right) 1_{\left\{x_{j}>0\right\}} \\
& -\sum_{i=1}^{N} \sum_{j=1}^{N} \mu_{i} \rho_{i} P_{i j}^{-} \beta_{i, j} 1_{\left\{x_{j}>0\right\}} \\
& -\sum_{i=1}^{N} \lambda_{i}^{-} \beta_{0, i} 1_{\left\{x_{i}>0\right\}} \\
& +\sum_{i=1}^{N} \sum_{j=1}^{N} \mu_{i} \rho_{i} P_{i j}^{-}\left(1-\beta_{i, j}\right) 1_{\left\{x_{j}=0\right\}}
\end{aligned}
$$

Now if we cancel the $\mu_{i}$ term on both sides we remain with:

$$
\begin{aligned}
\sum_{i=1}^{N}\left[\lambda_{i}^{+}+\right. & \left.\lambda_{i}^{-} \alpha_{0, i} 1_{\left\{x_{i}>0\right\}}+\lambda_{i}^{-} 1_{\left\{x_{i}=0\right\}} \beta_{0, i}\right]= \\
& \sum_{i=1}^{N} \Lambda_{i}^{-} 1_{\left\{x_{i}>0\right\}} \\
& +\sum_{i=1}^{N} \mu_{i} \rho_{i} d_{i} \\
& +\sum_{i=1}^{N} \lambda_{i}^{-} \alpha_{0, i} Q_{i, N+1} \rho_{i} \\
& +\sum_{i=1}^{N} \sum_{j=1}^{N} \mu_{i} \rho_{i} \rho_{j} P_{i j}^{-} Q_{j, N+1} \alpha_{i, j} \\
& +\sum_{i=1}^{N} \sum_{j=1}^{N} \mu_{i} \rho_{i} P_{i j}^{-}\left(1-\alpha_{i, j}\right) 1_{\left\{x_{j}>0\right\}} \\
& -\sum_{i=1}^{N} \sum_{j=1}^{N} \mu_{i} \rho_{i} P_{i j}^{-} \beta_{i, j} 1_{\left\{x_{j}>0\right\}} \\
& -\sum_{i=1}^{N} \lambda_{i}^{-} \beta_{0, i} 1_{\left\{x_{i}>0\right\}} \\
& +\sum_{i=1}^{N} \sum_{j=1}^{N} \mu_{i} \rho_{i} P_{i j}^{-}\left(1-\beta_{i, j}\right) 1_{\left\{x_{j}=0\right\}}
\end{aligned}
$$


and $[8]$ in (26), yielding:

$$
\begin{aligned}
\sum_{i=1}^{N}\left[\lambda_{i}^{+}+\right. & \left.\lambda_{i}^{-} 1_{\left\{x_{i}=0\right\}} \beta_{0, i}\right]= \\
& +\sum_{i=1}^{N} \mu_{i} \rho_{i} d_{i} \\
& +\sum_{i=1}^{N} \lambda_{i}^{-} \alpha_{0, i} Q_{i, N+1} \rho_{i} \\
& +\sum_{i=1}^{N} \sum_{j=1}^{N} \mu_{i} \rho_{i} \rho_{j} P_{i j}^{-} Q_{j, N+1} \alpha_{i, j} \\
& +\sum_{i=1}^{N} \sum_{j=1}^{N} \mu_{i} \rho_{i} P_{i j}^{-} 1_{\left\{x_{j}>0\right\}} \\
& -\sum_{i=1}^{N} \sum_{j=1}^{N} \mu_{i} \rho_{i} P_{i j}^{-} \beta_{i, j} 1_{\left\{x_{j}>0\right\}} \\
& -\sum_{i=1}^{N} \lambda_{i}^{-} \beta_{0, i} 1_{\left\{x_{i}>0\right\}} \\
& +\sum_{i=1}^{N} \sum_{j=1}^{N} \mu_{i} \rho_{i} P_{i j}^{-}\left(1-\beta_{i, j}\right) 1_{\left\{x_{j}=0\right\}}
\end{aligned}
$$

Using $1_{\left\{x_{i}>0\right\}}=1-1_{\left\{x_{i}=0\right\}}$ in the left-hand-side term and in [10], we group terms in the left-hand-side, eliminate [12], and modify [10], resulting in:

$$
\begin{aligned}
\sum_{i=1}^{N}\left[\lambda_{i}^{+}+\right. & \left.\lambda_{i}^{-} \beta_{0, i}\right]= \\
& +\sum_{i=1}^{N} \mu_{i} \rho_{i} d_{i} \\
& +\sum_{i=1}^{N} \lambda_{i}^{-} \alpha_{0, i} Q_{i, N+1} \rho_{i} \\
& +\sum_{i=1}^{N} \sum_{j=1}^{N} \mu_{i} \rho_{i} \rho_{j} P_{i j}^{-} Q_{j, N+1} \alpha_{i, j} \\
& +\sum_{i=1}^{N} \sum_{j=1}^{N} \mu_{i} \rho_{i} P_{i j}^{-} 1_{\left\{x_{j}>0\right\}} \\
& +\sum_{i=1}^{N} \sum_{j=1}^{N} \mu_{i} \rho_{i} P_{i j}^{-} \beta_{i, j} 1_{\left\{x_{j}=0\right\}} \\
& +\sum_{i=1}^{N} \sum_{j=1}^{N} \mu_{i} \rho_{i} P_{i j}^{-}\left(1-\beta_{i, j}\right) 1_{\left\{x_{j}=0\right\}}
\end{aligned}
$$

Using [10] and [13] we have:

$$
\begin{array}{rlr}
\sum_{i=1}^{N}\left[\lambda_{i}^{+}+\right. & \left.\lambda_{i}^{-} \beta_{0, i}\right]= \\
& +\sum_{i=1}^{N} \mu_{i} \rho_{i} d_{i} & {[2]} \\
& +\sum_{i=1}^{N} \lambda_{i}^{-} \alpha_{0, i} Q_{i, N+1} \rho_{i} & {[3]} \\
& +\sum_{i=1}^{N} \sum_{j=1}^{N} \mu_{i} \rho_{i} \rho_{j} P_{i j}^{-} Q_{j, N+1} \alpha_{i, j} & {[6]} \\
& +\sum_{i=1}^{N} \sum_{j=1}^{N} \mu_{i} \rho_{i} P_{i j}^{-} 1_{\left\{x_{j}>0\right\}} & {[8 *]} \\
& +\sum_{i=1}^{N} \sum_{j=1}^{N} \mu_{i} \rho_{i} P_{i j}^{-} 1_{\left\{x_{j}=0\right\}}
\end{array}
$$

and $[8 *]$ and $[13 *]$ simplify again, and using (1) and (2), the terms [2], [3], [6] are written as:

$$
\begin{aligned}
\sum_{i=1}^{N}\left[\lambda_{i}^{+}+\right. & \left.\lambda_{i}^{-} \beta_{0, i}\right]= \\
& +\sum_{i=1}^{N} \mu_{i} \rho_{i}\left[1-\sum_{j=1}^{N}\left(P_{i j}^{+}+P_{i j}^{-}\right)\right] \\
& +\sum_{i=1}^{N} \lambda_{i}^{-} \alpha_{0, i} \rho_{i}\left[1-\sum_{k=1}^{N} Q_{i, k}\right] \\
& +\sum_{i=1}^{N} \sum_{j=1}^{N} \mu_{i} \rho_{i} \rho_{j} P_{i j}^{-} \alpha_{i, j}\left[1-\sum_{k=1}^{N} Q_{i, k}\right] \\
& +\sum_{i=1}^{N} \sum_{j=1}^{N} \mu_{i} \rho_{i} P_{i j}^{-}
\end{aligned}
$$

This then simplifies to:

$$
\begin{aligned}
\sum_{i=1}^{N}\left[\lambda_{i}^{+}+\right. & \left.\lambda_{i}^{-} \beta_{0, i}\right]= \\
& +\sum_{i=1}^{N} \mu_{i} \rho_{i}\left[1-\sum_{j=1}^{N} P_{i j}^{+}\right] \\
& +\sum_{i=1}^{N} \lambda_{i}^{-} \alpha_{0, i} \rho_{i}\left[1-\sum_{k=1}^{N} Q_{i, k}\right] \\
& +\sum_{i=1}^{N} \sum_{j=1}^{N} \mu_{i} \rho_{i} \rho_{j} P_{i j}^{-} \alpha_{i, j}\left[1-\sum_{k=1}^{N} Q_{j, k}\right]
\end{aligned}
$$


We can now group the negative terms on the right-hand-side with $\left[\lambda_{i}^{+}+\lambda_{i}^{-} \beta_{0, i}\right]$ on the left-hand-side and write:

$$
\begin{array}{rlr}
\sum_{i=1}^{N} \Lambda_{i}^{+}= & & \\
& +\sum_{i=1}^{N} \mu_{i} \rho_{i} & {[2 * * *]} \\
& +\sum_{i=1}^{N} \lambda_{i}^{-} \alpha_{0, i} \rho_{i} & {[3 * *]} \\
& +\sum_{i=1}^{N} \sum_{j=1}^{N} \mu_{i} \rho_{i} \rho_{j} P_{i j}^{-} \alpha_{i, j} & {[6 * *]}
\end{array}
$$

Finally we use (4) to write:

$$
\sum_{i=1}^{N} \Lambda_{i}^{+}=\quad \sum_{i=1}^{N}\left[\mu_{i}+\Lambda_{i}^{-}\right] \rho_{i}
$$

completing the proof. Q.E.D. 